\title{
Nanophotonic modulators and photodetectors using silicon photonic and plasmonic device concepts
}

\author{
C. Koos ${ }^{\mathrm{a}, \mathrm{b},{ }^{*}}$, W. Freude ${ }^{\mathrm{a}}$, J. Leuthold ${ }^{\mathrm{c}}$, L. R. Dalton ${ }^{\mathrm{d}}$, S. Wolf ${ }^{\mathrm{a}}$, S. Muehlbrandt ${ }^{\mathrm{a}, \mathrm{b}}$, A. Melikyan ${ }^{\mathrm{b}}$, \\ H. Zwickel ${ }^{\mathrm{a}, \mathrm{b}}$, T. Harter ${ }^{\mathrm{a}, \mathrm{b}}$, Y. Kutuvantavida ${ }^{\mathrm{a}, \mathrm{b}}$, C. Kieninger ${ }^{\mathrm{a}, \mathrm{b}}$, M. Lauermann ${ }^{\mathrm{a}}$, D. Elder $^{\mathrm{d}}$ \\ ${ }^{a}$ Institute of Photonics and Quantum Electronics (IPQ), Karlsruhe Inst. of Technology (KIT), 76131 Karlsruhe, Germany \\ ${ }^{b}$ Inst. of Microstructure Technology (IMT), Karlsruhe Inst. of Techn. (KIT), 76344 Eggenstein-Leopoldshafen, Germany \\ ${ }^{\mathrm{c} N o w}$ with: Institute of Electromagnetic Fields (IEF), Swiss Federal Institute of Technology (ETH), Zurich, Switzerland \\ ${ }^{\mathrm{d}}$ University of Washington, Department of Chemistry, Seattle, WA 98195-1700, United States \\ *email: christian.koos@kit.edu
}

\begin{abstract}
Nanophotonic modulators and photodetectors are key building blocks for high-speed optical interconnects in datacom and telecom networks. Besides power efficiency and high electro-optic bandwidth, ultra-compact footprint and scalable co-integration with electronic circuitry are indispensable for highly scalable communication systems. In this paper, we give an overview on our recent progress in exploring nanophotonic modulators and photodetectors that combine the specific strengths of silicon photonic and plasmonic device concepts with hybrid integration approaches. Our work comprises electro-optic modulators that exploit silicon-organic hybrid ( $\mathrm{SOH}$ ) and plasmonic-organic hybrid $(\mathrm{POH})$ integration to enable unprecedented energy efficiency and transmission speed, as well as waveguide-based plasmonic internal photo-emission detectors (PIPED) with record-high sensitivities and bandwidths.
\end{abstract}

Keywords: Nanophotonic devices, modulators, photodetectors, silicon photonics, silicon-organic hybrid (SOH) integration, plasmonic-organic hybrid $(\mathrm{POH})$ integration, internal photoemission, plasmonic internal photoemission detectors (PIPED), optical interconnects

\section{SUMMARY}

From a technological point of view, silicon photonics represents an excellent integration platform, showing tremendous potential for large-scale photonic integration by exploiting mature foundry-based processes for fabless fabrication of photonic circuits and for co-integration with electronics [1]. From a functional point of view, however, silicon as an optical material falls short of distinct properties that are indispensable for a wide variety of devices. As a consequence, performance of all-silicon devices is often limited - in terms of speed, energy efficiency or device footprint. In this paper, we report on our recent progress in overcoming the functional limitations of the silicon photonic platform by combining conventional silicon photonic circuits with plasmonic devices and by hybrid integration of dedicated materials that complement the optical properties of silicon. We demonstrate the viability of the concepts by realizing electro-optic modulators with record performance parameters in terms of speed and energy efficiency as well as photodetectors with unprecedented electro-optic bandwidth. These devices might pave the path towards high-speed photonic-electronic signal processing at $\mathrm{THz}$ bandwidths.

For the fabrication of high-performance electro-optic modulators, we exploit the concept of silicon-organic hybrid $(\mathrm{SOH})$ integration that combines silicon photonic circuits with highly efficient organic electro-optic materials [2]. This approach overcomes the lack of second-order nonlinearities in bulk silicon and leads to highly efficient devices that clearly outperform conventional all-silicon depletion-type pn-modulators both in terms of speed and energy efficiency [2]. SOH devices have been demonstrated to exhibit voltage-length products down to $0.5 \mathrm{Vmm}$ and energy consumptions of only a few fJ per bit [3], [4]. The response of the electro-optic cladding materials is ultra-fast and enables small-signal modulation at frequencies beyond $100 \mathrm{GHz}$ [5] and generation of on-off keying (OOK) data signals at $100 \mathrm{Gbit} / \mathrm{s}$ [6]. We have further shown multi-level signaling at symbol rates of $64 \mathrm{GBd}$ and at operating temperatures of up to $80^{\circ} \mathrm{C}$ [7]. Moreover, we demonstrated generation of advanced modulation formats such as 16QAM at record- 
low energy consumptions and with symbol rates (bit rates) of up to $63 \mathrm{GBd}$ (252 Gbit/s) transmitted on a single wavelength and a single polarization [8] - [10]. We have further shown that the extraordinarily low drive voltage of SOH modulators allows operation of the devices directly from standard output ports of field-programmable gate arrays (FPGA), without external amplifiers or digital-to-analog converters. Such schemes can be used even if higher-order modulation formats such as 16QAM are to be generated [11]. The SOH approach is a versatile concept that goes far beyond electro-optic modulators. We have also demonstrated SOH lasers [13] that exploit doped light-emitting organic materials, as well as highly efficient SOH phase shifters with liquid crystals (LC) in the cladding [12].

Expanding upon our work on SOH devices, we have shown that the approach can also be transferred to plasmonic waveguide structures for electro-optic modulation [16] - [18]. While the length and hence the efficiency of these socalled plasmonic-organic hybrid ( $\mathrm{POH}$ ) modulators is limited by optical losses in the plasmonic waveguide section, the devices stand out due to their small footprint and their large electro-optic bandwidth [2]. We believe that POH devices may eventually open the route for modulation at $\mathrm{THz}$ frequencies [2].

Similarly, plasmonic device concepts allow to drastically reduce the footprint and to increase the bandwidth of photodetectors on the silicon photonic platform. We have recently demonstrated the concept of plasmonic internalphotoemission detectors (PIPED) that exploit photon-assisted transmission of hot carriers across potential barriers at metal-semiconductor interfaces [19]. Propagating surface plasmon polaritons (SPP) in metal-clad silicon waveguides are ideally suited for realizing such devices, as the plasmonic mode is strongly localized at the interface and hence perfectly concentrates the light to the region where absorption leads to the highest generation rate of photoelectrons. Our devices exploit a nanoscale silicon drift region of only $75 \mathrm{~nm}$ width, sandwiched between two metal layers with distinctively different light absorption characteristics and Schottky barrier heights. This leads to an ultra-narrow potential barrier and to short transit times under the influence of an external bias voltage. In a first proof-of-concept experiment, we demonstrated record-high sensitivities of more than $0.12 \mathrm{~A} / \mathrm{W}$ at a wavelength of $1550 \mathrm{~nm}$ along with large optoelectronic bandwidths well above $40 \mathrm{GHz}$ [19]. In a subsequent experiment, we have shown that PIPED can also be operated as photomixers for generation and homodyne reception of continuous-wave radiation at $\mathrm{THz}$ frequencies using monolithically integrated transmitter and receiver circuits on the silicon plasmonic platform [20]. We believe that $\mathrm{SOH}$ and POH modulators in combination with PIPED-based detection and photomixing schemes can pave the path towards large-scale integration of photonic-electronic circuits for processing of signals at $\mathrm{THz}$ frequencies.

\section{Acknowledgements}

This work was supported by the European Research Council (ERC Starting Grant 'EnTeraPIC', number 280145), by the Alfried Krupp von Bohlen und Halbach Foundation, by the EU-FP7 projects BigPipes and Phoxtrot, by the Helmholtz International Research School for Teratronics (HIRST), by the Karlsruhe School of Optics \& Photonics (KSOP), by the Erasmus-Mundus Joint Doctorate Program EUROPHOTONICS, and by the Karlsruhe Nano-Micro Facility (KNMF).

\section{REFERENCES}

[1] Sun, C. et al.: "Single-chip microprocessor that communicates directly using light," Nature 528, 534-538 (2015)

[2] Koos, C. et al.: "Silicon-organic hybrid (SOH) and plasmonic-organic hybrid (POH) integration,” J. Lightw. Technol. 24, 256268 (2016)

[3] Koeber, S. et al.: "Femtojoule electro-optic modulation using a silicon-organic hybrid device," Light: Science \& Applications $\mathbf{4}$, e255 (2015)

[4] Palmer, R. et al.: "High-speed, low drive-voltage silicon-organic hybrid modulator based on a binary-chromophore electro-optic material," J .Lightw. Technol. 32, 2726-2734 (2014)

[5] Alloatti, L. et al. "100 GHz silicon-organic hybrid modulator," Light: Science \& Applications 3, e173 (2014)

[6] Hartmann, W. et al. "100 Gbit/s OOK using a silicon-organic hybrid (SOH) modulator," 41th European Conf. Opt. Commun. (ECOC'15), Valencia, Spain, Sept. 27 - Oct. 5, 2015. Paper PDP1.4

[7] Lauermann, M. et al.: "Generation of $64 \mathrm{GBd} 4 \mathrm{ASK}$ signals using a silicon-organic hybrid modulator at $80^{\circ} \mathrm{C}$," Opt. Express 24 , 9389-9396 (2016)

[8] Wolf, S. et al. “An energy-efficient 252 Gbit/s silicon-based IQ-modulator,” Optical Fiber Communication Conference (OFC'16), Los Angeles (CA), USA, March 20-24, 2016, Paper Th3J.2

[9] Lauermann, M et al.: "40 GBd 16QAM Signaling at 160 Gbit/s in a Silicon-Organic Hybrid (SOH) Modulator," J. Lightw. Technol. 33, 1210-1216 (2015)

[10] Lauermann, M. et al. "Low-power silicon-organic hybrid (SOH) modulators for advanced modulation formats,” Opt. Express 22, 29927-29936 (2014)

[11] Wolf, S. et al. "DAC-less amplifier-less generation and transmission of QAM signals using sub-volt silicon-organic hybrid modulators," J. Lightw. Technol. 33, 1425-1432 (2015) 
This article has been accepted for publication in Proc. SPIE Physics and Simulation of Optoelectronic Devices XXV (OPTO-SPIE'17), San Francisco (CA), USA, Jan. 28 - Feb. 2 , paper 10098-6 (2017) (invited) DOI: http://dx.doi.org/10.1117/12.2256536

[12] Pfeifle, J. et al. "Silicon-organic hybrid phase shifter based on a slot waveguide with a liquid-crystal cladding," Opt. Express 20 (2012), 15359-15376

[13] Korn, D. et al.: "Lasing in silicon-organic hybrid (SOH) waveguides,” Nature Communications 7, 10864 (2016)

[14] Weimann, C. et al.: "Silicon-organic hybrid (SOH) frequency comb sources for terabit/s data transmission," Opt. Express 22, 3629-3637 (2014).

[15] Lauermann, M. et al.: "Integrated optical frequency shifter in silicon-organic hybrid (SOH) technology,” Opt. Express 24, 11694 - 11707 (2016)

[16] Melikyan, A. et al.: “High-speed plasmonic phase modulators,” Nature Photon. 8, 229-233 (2014).

[17] Haffner, C. et al.: "All-plasmonic Mach-Zehnder modulator enabling optical high-speed communication at the microscale," Nature Photonics 9, 525-528 (2015)

[18] Melikyan, A. et al. "Plasmonic-organic hybrid (POH) modulators for OOK and BPSK signaling at 40 Gbit/s," Opt. Express 23, 9938-9946 (2015)

[19] Muehlbrandt, S. et al.: "Silicon-plasmonic internal-photoemission detector for $40 \mathrm{Gbit} / \mathrm{s}$ data reception," Optica 3, 741-747 (2016)

[20] Harter, T. et al.: "Silicon-plasmonic photomixer for generation and homodyne reception of continuous-wave THz radiation," Conf. on Lasers and Electro-Optics (CLEO 2016), San Jose (CA), USA, June 5-10, 2016, Paper SM4E.5 